\title{
Pulmonales Hamartom - seltene Ursache für rezidivierenden pulmonalen Infekt unter Immunsuppression
}

\author{
Pulmonary Hamartoma - A Rare Cause of Recurrent Pneumonia
}

Autoren

Institute
H.-J. Seyfarth ${ }^{1}$, S. Hammerschmidt ${ }^{1}$, C. Gessner ${ }^{1}$, T. Gradistanac ${ }^{2}$, H. Wirtz ${ }^{1}$

Abteilung Pneumologie der Universität Leipzig (Leiter: Prof. Dr. med. H. Wirtz)

${ }^{2}$ Institut für Pathologie der Universität Leipzig (Direktor: Prof. Dr. med. C. Wittekind) eingereicht 2.5. 2006

$\begin{array}{ll}\text { akzeptiert 29.5. } 2006 & 0\end{array}$

\section{Bibliografie}

DOI $10.1055 / \mathrm{s}-2006-944236$

Pneumologie 2006; 60; 537-538

(c) Georg Thieme Verlag KG

Stuttgart · New York

ISSN 0934-8387

Korrespondenzadresse Dr. Hans-Jürgen Seyfarth Medizinische Universitätsklinik I Johannisallee 32, 04103 Leipzig seyh@medizin.uni-leipzig.de

Serienherausgeber

S. Ewig, Bochum

T. Schaberg, Rotenburg
Ein 49-jähriger Patient stellte sich mit Reizhusten, Belastungsdyspnoe und Fieber zu einer Nachsorgeuntersuchung 5 Monate nach Herztransplantation wegen ischämischer Kardiomyopathie vor. Bei klinisch und röntgenologisch gestütztem Verdacht auf einen bronchopulmonalen Infekt wurde unter Berücksichtigung der Immunsuppression eine antibiotische Therapie eingeleitet. Im Verlauf rezidivierte der Infekt, Reizhusten und Dyspnoe persistierten. Mit dieser Befundkonstellation wurde der Patient vorgestellt. Bei der Auskultation fiel ein in- und exspiratorischer Stridor über dem rechten Mittelfeld auf. Die Lungenfunktion zeigte eine Einschränkung der Vitalkapazität, eine relative Überblähung, verminderte endexspiratorische Flüsse und einen erhöhten Atemwegswiderstand bei Ruheatmung. Laborchemisch war neben einer Anämie und erhöhten Retentionsparametern ein C-reaktives Protein von $38 \mathrm{mg} / \mathrm{l}$ auffällig. Im nativ durchgeführten Computertomogramm des Thorax stellte sich eine nach dorsal konvexe, sichelförmige Einengung des Unterlappenbronchus rechts dar (Obb. 1).

Während der Bronchoskopie fand sich als Korrelat der Bildgebung ein polypöser Tumor, der den rechten Unterlappenbronchus nahezu komplett verschloss ( Abb. 2a). Die Ostien zum Mittellappen und zu RB6 erschienen unbeeinträchtigt. Die histologische Aufarbeitung der Biopsie des Polypen erbrachte keinen Hinweis für Malignität - Bronchialschleimhaut mit chronischer Entzündung und einer Plattenepithelmetaplasie wurde beschrieben. Es bestand der Verdacht, dass es sich bei diesem endobronchialen Tumor um einen Granulationsgewebspolypen handelt.

Daraufhin wurde in einer zweiten Bronchoskopie nach Sedierung und Intubation der Polyp mit dem Nd-YAG-LASER im contact mode (Einzelenergie 5 Watt, Gesamtenergie 270 Watt) herausgeschnitten ( $\mathbf{A b b} \mathbf{2} \mathbf{2 b}, \mathbf{c})$. In der histologi-

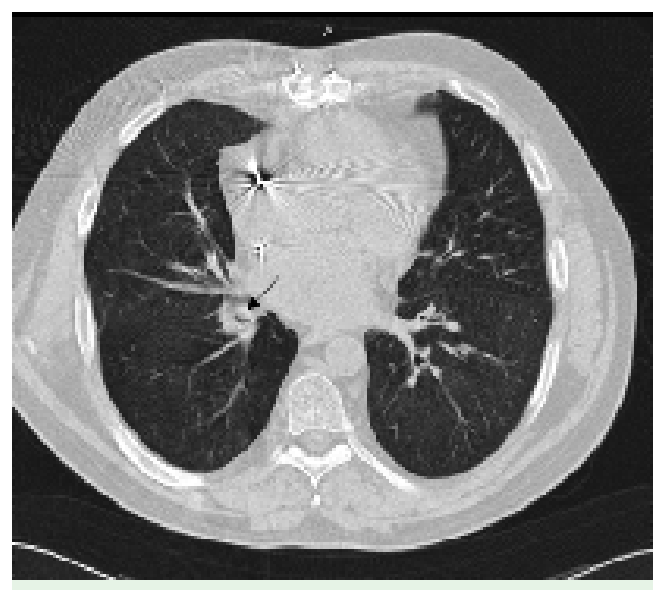

Abb. 1 Computertomographie des Thorax.

schen Untersuchung stellte sich der in toto geborgene Polyp als pulmonales Hamartom dar ( Abb. 3a, b). Die bronchoskopische Kontrolluntersuchung wenige Tage später zeigte bereits eine reizlose, glatte Abtragungsstelle ( Abb. 2d). Die Beschwerden sistierten, die Lungenfunktion besserte sich.

Das pulmonale Hamartom ist der häufigste der insgesamt seltenen benignen pulmonalen Tumoren. Die endobronchiale Manifestation ist eine Rarität. Ein multilokuläres Vorkommen ist möglich [1,2]. Die Symptome reichen von Reizhusten über Dyspnoe und rezidivierende Infekte bis hin zu Hämoptysen [3]. Die wenigen in der Literatur beschriebenen, ähnlich gelagerten Fälle mündeten häufig in eine Lobektomie [4,5]. Retrospektiv ist der Polyp in der präoperativen CT zu sehen, die Frage, wie lange das pulmonale Hamartom bereits in dieser Ausprägung bestand, muss offen bleiben. Die Bronchoskopie gehört nicht zu den vorbereitenden Untersuchungen vor einer Herztransplantation, sollte aber bei unklarer respiratorischer Symptomatik durchgeführt werden. 


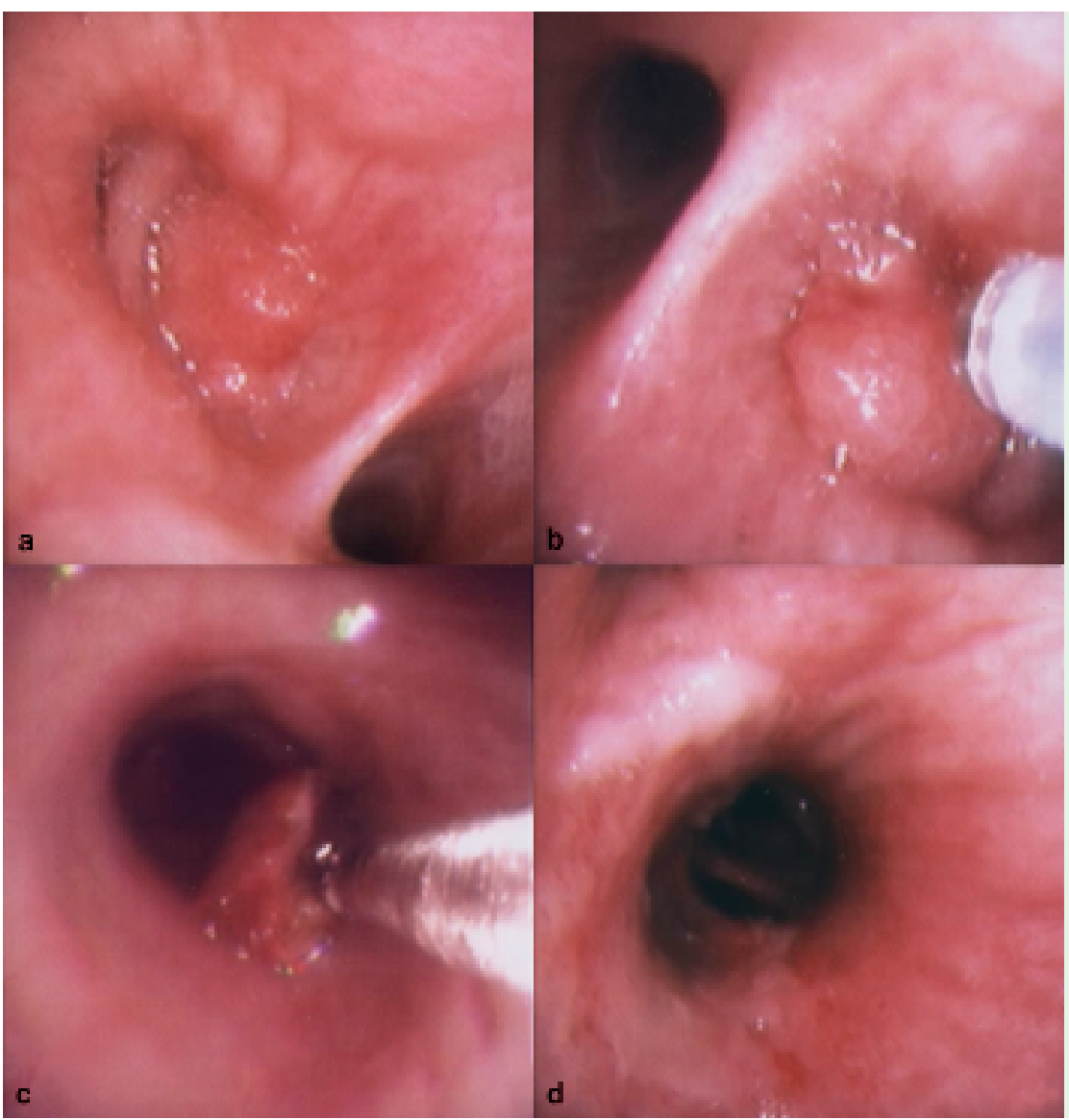

Abb. 2 Endobronchialer Befund vor der Abtragung des Polypen (a); mit LASER-Faser (b); Entfernung des Polypen in toto - Zange mit Polyp hier in der Trachea (c); Aspekt 3 Tage nach Abtragung des Polypen (d).

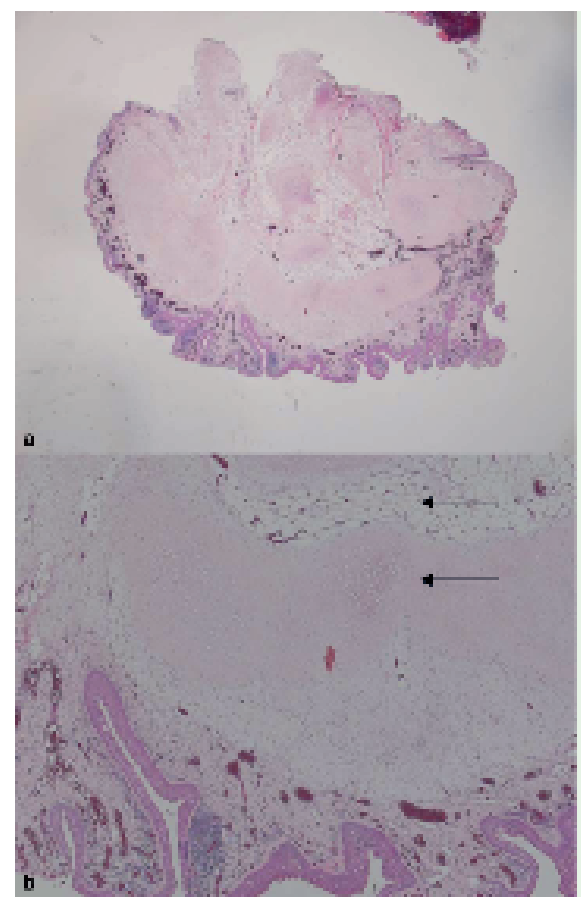

Abb. 3 Histologische Darstellung: des gesamten pulmonalen Hamartoms (a) eines Ausschnittes (b, Pfeile: Fettgewebe bzw. Knorpel).

\section{Literatur}

1 Ribet M, Jaillard-Thery S, Nuttens MC. Pulmonary hamartoma and malignancy. J Thorac Cardiovasc Surg 1994; 107: 611-614

2 Laroche CM, Stewart S, Wells F et al. Multiple recurrent intrapulmonary and endobronchial mesenchymomas (hamartomas). Thorax 1993; 48: $572-573$

3 Sharkey RA, Mulloy EM, O'Neil S. Endobronchial hamartoma presenting as massive haemoptysis. Eur Respir J 1996; 9: 2179-2180

4 Kurkcuoglu IC, Demircan S, Kurul IC et al. Endobronchial lipomatous hamartoma. Asian Cardiovasc Thorac Ann 2005; 13: 372 - 373

5 Yilmaz S, Ekici A, Erdogan S et al. Endobronchial lipomatous hamartoma: CT and MR imgaging features (2004:5b). Eur Radiol 2004; 14 : $1521-1624$ 